\title{
THE ROLE OF DENTAL STEM CELLS IN REGENERATION
}

\author{
MONICA ANGELA MAXIM ${ }^{1}$, OLGA SORITAU ${ }^{2}$, MIHAELA BACIUT ${ }^{3}$, \\ SIMION BRAN ${ }^{3}$, GRIGORE BACIUT ${ }^{3}$
}

\author{
${ }^{1}$ Iuliu Hatieganu University of Medicine and Pharmacy, Cluj-Napoca, Romania \\ ${ }^{2}$ Prof. Dr. Ion Chiricuta Institute of Oncology, Cluj-Napoca, Romania \\ ${ }^{3}$ Department of Cranio-Maxillofacial Surgery and Dental Emergencies, Iuliu \\ Hatieganu University of Medicine and Pharmacy, Cluj-Napoca, Romania
}

\begin{abstract}
Mesenchymal stem cells (MSCs) are adult stem cells that have the capacity of rising multiple cell types.

A rich source of mesenchymal stem cells is represented by the dental tissues: the periodontal ligament, the dental pulp, the apical papilla, the dental follicle and the deciduous teeth.

The aim of this review is to characterize the main dental-derived mesenchymal stem cell population, and to show their important role in tissue regeneration based on their properties : the multi-potency, the high proliferation rate, the differentiation in multiple cell lineages, the high cell viability and the positive expression for mesenchymal cell markers.

Tissue regeneration or de novo'formation of craniofacial structures is the future of regenerative medicine, offering a solution for congenital malformations, traumas and other diseases.
\end{abstract}

Keywords: dental stem cells, tissue regeneration, characterization, mesenchymal stem cells, tissue engineering

\section{Introduction}

The mesenchymal stem cells have an expanded distribution in adult tissues, including those of dental origin.

In the last few years the regenerative medicine has gained a lot of interest based on special stem cells characteristics like replacing, maintaining, repairing and enhancing tissue function [1].

The term "tissue engineering" was first used by Langer and Vacanti in 1993 and it described tissue regeneration by cell transplantation with or without a scaffold for cellular growth [2].

Mesenchymal stem cells are characterized by two major properties : the auto-renewal capacity and the differentiation into multiple cell lineages like bone cells, adipose cells and cartilage cells [3].

Manuscript received: 20.05.2015

Received in revised form: 27.07.2015

Accepted: 20.08.2015

Address for correspondence: Maxim.Monica@umfcluj.ro
In the past decade, the mesenchymal stem cells have been an ideal candidate used to repair and/or regenerate defective organs and tissues like bone, cartilage, spinal cord and heart [4].

A new source of mesenchymal stem cells is represented by the dental tissues.

Stem cells have been successfully isolated from the dental pulp, the periodontal ligament, the dental follicle, the apical papilla and from the deciduous teeth $[5,6]$.

The human body tissues have regeneration capacities during life, such as the epithelial tissue that can recover if damaged, and the connective tissue, the bone and the cartilage that can recover in case of minor damage $[7,8]$.

The tissue regeneration techniques are also found in dental medicine, in processes that involve the tertiary dentin and the guided tissue regeneration of the periodontium. Researchers are interested in using the multi-potent stem cells to develop a whole tooth with all its complexity $[9,10]$.

The tooth is a complex organ formed by soft and 


\section{Dental Medicine}

hard tissues. The hard tissues are represented by the dentin which is covered by the enamel at the crown level and by the cement at the root level. The soft tissues are represented by the dental pulp, a highly vascularized and innervated tissue, and the periodontal ligament which is a complex structure that maintains the organ in the tooth socket $[1,10]$.

The aim of the present review is to characterize and to describe the current knowledge concerning the mesenchymal dental stem cells with their potential role in regeneration.

\section{Mesenchymal stem cells from the dental pulp:}

The dental pulp is a connective tissue. It consists of many cell types, and it is sheltered in a space with rigid walls in case of tissue integrity [10].

The dental pulp is composed of four layers:

- The external layer, formed by odontoblasts. It's main role is to form the dentin that protects the pulp of the external stimulus.

- An acellular layer, represented by the extracellular matrix.

- A layer of progenitor cells, with multi-potent characteristics.

- The internal layer, represented by the arterial and the nervous system of the pulp, which explains the high sensitivity of the pulp [11].

This new source of stem cells was discovered by Gronthos et al in 2000 isolating stem cells from the impacted third molars [12,13].

These cells have been named Dental Pulp Stem Cells (DPSC) and they are characterized by a high proliferation rate and cellular differentiation depending on the medium culture [3].

They are able to maintain their self-renewal, and to form pulp-like tissue, bone-like tissue, odontoblast-like cells if transplanted in animal models, which indicates their important role in regenerative cell based therapy [12].

DPSCs express the mesenchymal and bone marrow stem cell markers (CD146, STRO-1) and the embryonic stem cell marker (Oct4).

The microenvironment of the dental pulp is auspicious for the stem cells, because of the lack of oxygen and nutrients, due to the low diameter of the apical foramen $[14,15]$.

\section{Mesenchymal stem cells from the apical papilla}

The apical papilla is one of the soft tissues of the dental organ, represented by specialized connective tissue placed at the tooth root apex [16].

This is a new harvesting zone, and the harvested stem cells, called SCAP are characterized by a highly proliferation rate, that makes them suitable for forming odontoblast-like cells for a cell-based regeneration, being able to produce dentin in vivo and to complete the root formation [17].

As other dental stem cells, SCAPs expressed the early mesenchymal surface markers like STRO-1 and
CD146, and also expressed CD24, a unique marker for this cell population [1].

Mesenchymal stem cells from the deciduous teeth

This cells have been named SHED (stem cells from the deciduous teeth) and are isolated from the remaining dental pulp [18].

The isolated stem cells have osteoinductive capacities, and multi-differentiation in mesenchymal cells and a higher number of colony forming cells than DPSCs [3].

These stem cells can produce dentin and are very important in neurodegenerative diseases by their secretion of neurotrophic factors, being useful in neuronal regeneration [18].

SHEDs were found to express early mesenchymal stem cell markers (Stro-1) and embryonic stem cell markers (Oct4, Nanog) that may suggest a neural crest origin of these cells [19].

\section{ligament}

Mesenchymal stem cells from the periodontal

The periodontal ligament is a soft connective tissue which lies between the root cement and the dental alveola [20].

It is formed by many cellular types, including mesenchymal undifferentiated cells with osteoinductive role and by extracellular components (collagen fibres). The main role of the collagen fibers is to maintain the tooth in the alveolar bone and to distribute the masticatory forces [21].

The undifferentiated mesenchymal cells are called "stem-like cells" with osteoinductive role, and have been named PDLSC. They have multi-differentiation capacities, into bone cells, adipose cells, and cartilage and in connective tissue rich in collagen. The main role of this stem cells is to maintain homeostasis and to regenerate the periodontium $[1,22]$.

This cell population presents a high rate of positivity (70-90\%) for mesenchymal stem cell marker proteins, such as CD44, CD73, CD92, CD105 and absence for the hematopoietic markers, like CD34, CD45. They show high expression for embryonic markers too, like Nanog, Sox2, SSEA4. This indicates the mesenchymal origin of this cells, and the pluri-potency characteristics $[4,16]$.

The correct manipulation of the stem cells has a major role in tissue engineering, suggesting that these cells could be used for the novo formation of a dental root as an implant for an artificial dental crown [3,20].

Mesenchymal stem cells from the dental follicle

The dental follicle is the soft connective tissue that surrounds the not yet erupted dental organ [3].

During tooth eruption this connective tissue transforms into the periodontal tissue that surrounds the tooth, becoming visible in the oral cavity [1].

During dental eruption, this tissue is behaving like a multi-potent tissue that generates the root cement, the 
periodontal ligament and the bone. We can say that the cells from the dental follicle have resembling characteristics as mesenchymal stem cells through their capacity to attach in cell culture and the differentiation into multiple cell lineages, being named DFPC $[2,11,23]$.

These cells were first isolated in 2005 from the third molar by Morsczeck et al, using the same method as DPSC. The cells were fibroblast-like and expressed various markers, like neural cell marker expression and cementoblast markers. In vitro studies demonstrated their capacity to differentiate into osteoblasts, adypocites and neuronal cells $[1,24]$.

\section{Conclusions}

Tissue engineering is based on creating favorable conditions to regenerate the damaged/lost tissues.

The future is the regeneration of whole organs and complex systems, starting from an initial stem cell line, molecules and innovative scaffolds.

Because of the high frequency of the periodontal diseases caused by the loss of the connective tissue attachment and the loss of the alveolar bone, the number of patients with tooth loss problems has increased. That is why the researchers are trying to find new ways to form new alveolar bone and periodontal ligament.

The difficulty of the "guided tissue regeneration" technique with limited success and results in time requires the development of a new regeneration therapy based on mesenchymal stem cells.

Due to their characteristics, like the high proliferation rate, the multi-differentiation and the presence of the stemness markers, mesenchymal stem cells become an attractive target in tissue engineering.

Further extensive studies need to be performed in order to create suitable scaffolds for periodontium regeneration and for more appropriate approaches for tissue engineering in dental practice.

\section{Acknowledgement}

Dr. Maxim Monica Angela acknowledges financial support from an POSDRU grant no. 159/1.5/S/138776/ grant with title: Model colaborativ institutional pentru translatarea cercetarii stiintifice biomedicale in practica clinica - TRANSCENT [Institional collaborative model for the translation of biomedical research into clinical practice].

\section{References}

1. Jamal M, Chogle S, Goodis H, Karam SM. Dental stem cells and thier potential role in regenerative medicine. J Med Sci. 2011;4:53-61.

2. Honda MJ, Imaizumi $\mathrm{M}$, Tsuchiya $\mathrm{S}$, Morsczeck $\mathrm{C}$. Dental follicle stem cells and tissue engineering. J Oral Sci. 2010;52(4):541-552.

3. Estrela C, Alencar AH, Kitten GT, Vencio EF, Gava E. Mesenchymal stem cells in the dental tissues: perspectives for tissue regeneration. Braz Dent J. 2011;22(2):91-98.
4. Xiao L, Nasu M. From regenerative dentistry to regenerative medicine: progress, challenges, and potential applications of oral stem cells. Stem Cells Cloning. 2014;7:89-99.

5. Suma GN, Arora MP, Lakhanpal M. Stem cell therapy: A novel treatment approach for oral mucosal lesions. J Pharm Bioallied Sci. 2015;7(1):2-8.

6. Liu Y, Hu J, Wang S. Mesenchymal stem cell-mediated treatment of oral diseases. Histol Histopathol. 2014;29(8):10071015 .

7. Bojic S, Volarevic V, Ljujic B, Stojkovic M. Dental stem cells-characteristics and potential. Histol Histopathol. 2015;29(6):699706.

8. Machado E, Fernandes MH, Gomes Pde S. Dental stem cells for craniofacial tissue engineering. Oral Surg Oral Med Oral Pathol Oral Radiol. 2012;113(6):728-733.

9. Liu J, Yu F, Sun Y, Jiang B, Zhang W, Yang J, et al. Concise reviews: Characteristics and potential applications of human dental tissue-derived mesenchymal stem cells. Stem Cells. 2015;33(3):627-638.

10. Graziano A, d'Aquino R, Laino G, Papaccio G. Dental pulp stem cells: a promising tool for bone regeneration. Stem Cell Rev. 2008;4:21-26.

11. Saito MT, Silvério KG, Casati MZ, Sallum EA, Nociti FH Jr. Tooth-derived stem cells: Update and perspectives. World J Stem Cells. 2015;7(2):399-407.

12. Han J, Menicanin D, Gronthos S, Bartold PM. Stem cells, tissue engineering and periodontal regeneration. Aust Dent J. 2014;59 Suppl 1:117-130.

13. Gronthos S, Arthur A, Bartold PM, Shi S. A method to isolate and culture expand human dental pulp stem cells. Methods Mol Biol. 2011;698:107-121.

14. Padial-Molina M, O’Valle F, Lanis A, Mesa F, Dohan Ehrenfest DM, Wang HL, et al. Clinical Application of Mesenchymal Stem Cells and Novel Supportive Therapies for Oral Bone Regeneration. Biomed Res Int. 2015;2015:341327.

15. Tatullo M, Marrelli M, Paduano F. The regenerative medicine in oral and maxillofacial surgery: the most important innovations in the clinical application of mesenchymal stem cells. Int J Med Sci. 2015;12(1):72-77.

16. Zhao N, Wu Z, Qin L, Guo Z, Li D. Characteristics and Tissue Regeneration Properties of Gingiva-Derived Mesenchymal Stem Cells. Crit Rev Eukaryot Gene Expr. 2015;25(2):135-144.

17. Hemmat S, Lieberman DM, Most SP. An introduction to stem cell biology. Facial Plast Surg. 2010;26(5):343-349.

18. Kashyap R. SHED - Basic Structure for Stem Cell Research. J Clin Diagn Res. 2015;9(3):ZE07-ZE09.

19. Miura M, Gronthos S, Zhao M, Lu B, Fisher LW, Robey PG, et al. SHED: stem cells from human exfoliated deciduous teeth. Proc Natl Acad Sci U S A. 2003;100(10):5807-5812.

20. Hynes K, Menicanin D, Gronthos S, Bartold PM. Clinical utility of stem cells for periodontal regeneration. Periodontol 2000. 2012;59(1):203-227.

21. Moshaverinia A, Xu X, Chen C, Ansari S, Zadeh HH, Snead ML, et al. Application of stem cells derived from the periodontal ligament or gingival tissue sources for tendon tissue regeneration. Biomaterials. 2014;35(9):2642-2650.

22. Mayo V, Sawatari Y, Huang CYC, Garcia-Godoy F. Neural crest-derived dental stem cells-where we are and where we are going. J Dent. 2014;42(9):1043-1051.

23. Morsczeck C, Götz W, Schierholz J, Zeilhofer F, Kühn U, Möhl C, et al. Isolation of precursor cells (PCs) from human 


\section{Dental Medicine}

dental follicle of wisdom teeth. Matrix Biol. 2005;24:155-165.

24. Kon E, Filardo G, Roffi A, Di Martino A, Hamdan M, De
Pasqual L, et al. Bone regeneration with mesenchymal stem cells. Clin Cases Miner Bone Metab. 2012;9(1):24-27. 